\title{
Factores de resiliencia en niños y adolescentes con cáncer y su relación con el género y la edad
}

\author{
Sheila Pintado* y María Fernanda Cruz*
}

Recepción: 10 de marzo de 2016

Aceptación: 23 de junio de 2016

*Universidad de las Américas Puebla, México.

Correos electrónicos: maria.pintado@udlap.mx; maria. cruzar@udlap.mx

Se agradecen los comentarios de los árbitros de la revista.

(c) $\$ \cong$

\begin{abstract}
Resumen. Se analizan los aspectos de la resiliencia predominantes en una muestra de 54 niños y adolescentes con cáncer para determinar si existen diferencias en función del género y la edad mediante una entrevista clínica y el Inventario de Factores Personales de Resiliencia. Los resultados mostraron que el sentido del humor es el factor de resiliencia predominante. Además, se encontró que los hombres tienen niveles más elevados en sentido del humor, autonomía, empatía y autoestima y las mujeres en creatividad. Respecto a la edad, los niños mayores presentaron niveles más elevados de autoestima, empatía y autonomía. En sentido del humor y creatividad no se encontraron diferencias significativas.
\end{abstract}

Palabras clave: resiliencia, cáncer, adolescencia, niñez.

\begin{abstract}
Resilience Factors in Children and Adolescents with Cancer and the Relationship with Gender and Age

Abstract. The objective of this study was to analyze the prevailing aspects of the resilience in a sample of 54 children and adolescents with cancer, and to determinate if there are differences according to the gender and age, through a clinical interview and the inventory of personal factors of resilience.

The results showed that the sense of humor was the most prevalent factor in the sample. Moreover, differences between men and female were observed: men had higher levels of sense of humor, autonomy, empathy and self-esteem; and women had higher levels of creativity. In relation to the age, resilience was higher in older children in self-esteem, empathy and autonomy. However, there were not differences in sense of humor and creativity.
\end{abstract}

Keywords: resilience, cancer, adolescence, childhood.

\section{Introducción}

En las últimas décadas ha aumentado el interés en el estudio de la resiliencia. Se han podido observar muchos casos de niños que han pasado por situaciones difíciles y traumáticas en su infancia y que no desarrollan problemas de salud mental, consumo de drogas o conductas criminales posteriores, sino que por el contrario presentan funciones y procesos adaptativos (Becoña, 2006; Masten y Reed, 2002; Romero, 1998; Tarragona, 2011).
Debido a esto, muchos terapeutas se preguntan qué características o factores están asociados a esta capacidad resiliente y cómo se podría trabajar sobre ellas y aumentarlas en beneficio de los pacientes. Es decir, por qué algunos niños, pese a las situaciones adversas, traumas graves y amenazas contra su desarrollo logran salir adelante y desarrollarse de forma positiva (Muñoz, 2005).

Cada año se les diagnostica cáncer a más de 160000 niños en el mundo. La Sociedad Americana contra el Cáncer en 2012 concluyó que la curación infantil se encuentra en 80\% 
de países desarrollados, lo cual demuestra que esta enfermedad no necesariamente termina en muerte. Asimismo, se ha visto que un diagnóstico temprano, la correcta atención al enfermo, las relaciones de apoyo y los pensamientos y emociones positivos del paciente son fundamentales para superar la enfermedad (Cadena et al., 2013).

La Sociedad Internacional de Oncología Pediátrica (SIOP) y la Asociación Americana de Pediatría (AAP) coinciden en que el niño y adolescente a los que se les diagnostica cáncer responden a ciertas características establecidas de su edad, por lo que deben tomarse en cuenta de acuerdo con sus demandas médicas, psicológicas, emocionales, educativas y sociales en relación con su desarrollo (Chaves, 2014).

En 2011 el Instituto Mexicano del Seguro Social (IMSs) realizó un registro sobre las causas de muerte en niños mexicanos. En él sobresale un cambio significativo, ya que el resultado demostró que el cáncer es la segunda causa de muerte en niños hasta los 14 años.

Esto ha promovido el cáncer infantil como un problema de salud pública. Las personas que pasan por esa enfermedad necesitan de fortalezas psicológicas y sociales para afrontar los altibajos presentes en dicho padecimiento, como la capacidad resiliente (Cedillo, 2014).

El cáncer es una enfermedad crónica que pone en riesgo la vida. El sufrimiento es para la persona que lo padece y para su familia (Mystakidou et al., 2006). La persona con cáncer presenta síntomas de la enfermedad y de su tratamiento, como náuseas, vómitos, pérdida de peso o fatiga; de igual forma, se ve afectada por daños psicológicos.
Respecto a los niños, el cáncer les genera una amplia lista de emociones como miedo, ira, soledad, depresión o ansiedad. Los más pequeños manifiestan preocupación por el dolor y miedo de ser separados de sus padres y entorno cuando se les tiene que hospitalizar (Bragado, 2010; Espada et al., 2004). Por todo ello, se desarrolla el trabajo con la resiliencia como una corriente de la psicología positiva para promover la salud mental en estos niños (Álamo et al., 2012).

La resiliencia en pacientes con cáncer es un aspecto que fortalece la salud mental y proporciona herramientas para una mejor adaptación incluso, a un nivel superior respecto a cómo vivían antes (Bowen et al., 2003; Castro y MorenoJiménez, 2007; García, 2013; Vázquez y Castilla, 2007).

El término resiliencia tuvo su origen en la física y hacía referencia a la capacidad de un cuerpo de resistir, permanecer fuerte y no deformarse. Posteriormente, fue adaptado en ciencias sociales y de la salud como la capacidad de permanecer, crecer y salir con éxito de cualquier adversidad (Rutter, 1992). Y desde ese momento, varios autores han sugerido diferentes definiciones para este concepto, las cuales se enfocan en cómo los niños y jóvenes se desarrollan al momento de afrontar circunstancias problemáticas en las que se valorizan los aspectos positivos de la adaptación (Masten y Powell, 2003; Stein et al., 2000). Para ilustrar mejor, en la tabla 1 se condensan algunas definiciones de resiliencia propuestas en las últimas décadas.

Con lo anterior, se entiende que existen diferentes definiciones para el concepto de resiliencia debido a que cada autor

\begin{tabular}{|c|c|}
\hline Autores & Definición de resiliencia \\
\hline Cabanyes (2010) & Capacidad de recuperación de un individuo ante situaciones traumáticas extremas. \\
\hline Manciaux (2003) & $\begin{array}{l}\text { Capacidad de una persona o grupo para seguir proyectándose en el futuro a pesar de acontecimientos desestabi- } \\
\text { lizadores y condiciones de vida difíciles. }\end{array}$ \\
\hline Cerezo (2014) & $\begin{array}{l}\text { Capacidad de resistir y recomponer las dificultades de la vida, de recuperarse y obtener una conducta adaptativa } \\
\text { después de la adversidad. }\end{array}$ \\
\hline Suárez y Krauskorpf (1995) & $\begin{array}{l}\text { Combinación de factores que van a permitir que un ser humano afronte y supere los problemas y dificultades de } \\
\text { su vida. }\end{array}$ \\
\hline Bonanno (2004) & Capacidad de responder a un evento traumático de forma positiva y después poder funcionar correctamente. \\
\hline $\begin{array}{l}\text { Manciaux, Vanistendael, Lecomte y } \\
\text { Cyrulnik (2001) }\end{array}$ & $\begin{array}{l}\text { Capacidad de una persona o grupo para seguir proyectándose en el futuro a pesar de acontecimientos desestabi- } \\
\text { lizadores, de condiciones de vida difíciles y de traumas a veces graves. }\end{array}$ \\
\hline Cadena, Castro y Franklin (2013) & $\begin{array}{l}\text { Capacidad o conjunto de fortalezas que tienen las personas para continuar visualizándose en un futuro, aun- } \\
\text { que existan situaciones devastadoras, condiciones de vida difíciles o experiencias traumáticas. }\end{array}$ \\
\hline Puig y Rubio (2011) & $\begin{array}{l}\text { Proceso que se origina a partir de que una persona se enfrenta a una dificultad, desarrolla sus habilidades } \\
\text { personales y encuentra apoyo en los recursos y personas de su alrededor para poder superar la situación. }\end{array}$ \\
\hline Grotberg (1995) & $\begin{array}{l}\text { Capacidad humana universal para hacer frente a las adversidades de la vida, superarlas o incluso ser transfor- } \\
\text { mado por ellas. }\end{array}$ \\
\hline Melillo, Suárez y Rodríguez (2004) & $\begin{array}{l}\text { Conjunto de procesos sociales e intrapsíquicos que posibilitan acceder al bienestar psicofísico a pesar de las } \\
\text { adversidades. }\end{array}$ \\
\hline
\end{tabular}


le otorga su propio enfoque. Sin embargo, la mayoría coincide en que este término es una herramienta utilizada por ciertas personas para superar positivamente alguna experiencia difícil o situación adversa que sin la capacidad de resiliencia podría llegar a ser traumática.

Como menciona Fraser et al. (1999), a pesar de las diferentes definiciones del concepto, la mayoría se diferencia por la teoría que las origina y las situaciones en que se aplica. Según estos autores, existen características que se comparten entre ellas como experimentar éxito después de exponerse a una situación de riesgo, ser capaz de adaptarse a las dificultades sosteniendo las competencias personales y obtener consecuencias positivas después de la crisis.

Por otra parte, también se han estudiado los elementos que componen la resiliencia. Algunos son una alta autoestima y autoconcepto positivo, habilidad de autorreflexión, capacidad para pensar críticamente y de realizar acciones de manera independiente, habilidades para solucionar problemas y adaptabilidad social, entre otros (Masten y Powell, 2003; Place et al., 2002).

Estos elementos promoverían el desarrollo de las personas al servir como apoyo ante situaciones difíciles o de riesgo ayudando a que el individuo no tenga una influencia negativa ni impidiendo su progreso (Saavedra y Villalta, 2008).

Por su parte, Grotberg (2006) propone tres dimensiones de la resiliencia: Yo soy, que incluye las cualidades relacionadas con la fortaleza interna de la persona, Yo puedo, o capacidades sociales y relaciones interpersonales, y Yo tengo, que se basa en relaciones de confianza y apoyo social percibido.

Siguiendo este planteamiento, Cáceres, Fontecilla y Kotliarenco (citados en González-Arratia, 2009) indican que las personas resilientes poseen las siguientes características: mayor coeficiente intelectual y habilidades para resolver problemas, mejores estilos de afrontamiento, motivación al logro, autonomía, autoestima y locus de control interno, empatía, conocimiento y manejo adecuado de relaciones interpersonales, voluntad y capacidad de planificación y sentido del humor.

De igual forma, hay estudios que demuestran que las personas resilientes hacen uso de las emociones positivas como estrategia de afrontamiento, tales como el sentido del humor, la creatividad y el pensamiento optimista (Fredrickson y Tugade, 2003). Salgado (2005) asegura que la autoestima, la autonomía, la empatía, el sentido del humor y la creatividad son características de la resiliencia de niños y niñas. Para Bragado (2010), entre las variables que se relacionan con la resiliencia, resaltan la esperanza, las expectativas positivas, la confianza y la autoestima, además de la cohesión, la adaptabilidad familiar y una comunicación abierta.
Dentro del estudio de la resiliencia, ha habido controversia en torno a la relación entre la resiliencia, la edad y el género. Hay algunos autores que postulan que existen diferencias en resiliencia en función de la edad. En ellas se observa que los niños más pequeños presentan mayores niveles de resiliencia (Bragado, 2010). Sin embargo, otros autores no han encontrado tales diferencias.

En cuanto al género, algunas investigaciones mostraron que no existen diferencias significativas al respecto. Sólo se encontraron diferencias en la variable de interacción (capacidad de tener relaciones íntimas y satisfactorias con otras personas) a favor de las mujeres (Prado y Águila, 2003). Por el contrario, en otras investigaciones sí se observaron diferencias en cuanto a los niveles de resiliencia totales, e incluso se postula que ser mujer sería una variable protectora y mayormente asociada a la resiliencia (González-Arratia et al., 2008; Morales y Díaz, 2011).

Asimismo, Grotberg (1995) concuerda con lo anterior e indica que el género es una variable que presenta diferencias en resiliencia. Recalca que los niños se sienten más inseguros, inhibidos y con dificultades de comunicación; en cambio, las niñas son más flexibles y dependientes de las figuras de apego, lo cual genera que en la resolución de conflictos tengan una mejor habilidad interpersonal y fortalezas internas para una mayor capacidad de afrontar la adversidad.

En investigaciones donde se analiza la resiliencia en pacientes con cáncer, se muestra que en niños diagnosticados al compararse con otros de la misma edad sin este diagnóstico presentan los mismos niveles en cuanto a síntomas de ansiedad y depresión. Se observa así un buen ajuste psicológico ante la enfermedad y los tratamientos (Allen et al., 1997; Dejong y Fombonne, 2006; Eiser et al., 2000; González-Arratia et al., 2009; Grootenhuis y Last, 2001; Noll et al., 1999; Von Essen et al., 2000). Phipps (2007) menciona que es importante considerar el cáncer en niños como una oportunidad para investigar sobre la resiliencia e incorporar modelos de la psicología positiva.

En un estudio donde se examinaron las características resilientes entre madres que tienen hijos con cáncer entre 8 y 17 años de edad, se observó que la familia es un elemento protector indispensable para que el niño desarrolle la capacidad de resiliencia (González-Arratia et al., 2011).

Por otro lado, en una investigación donde se analizaron los recursos que utiliza el adolescente con cáncer para sobrellevar la enfermedad, se vio que la capacidad de aceptar, vivir y confrontar el proceso de la enfermedad eran capacidades resilientes; además de otorgarle a la enfermedad una respuesta alternativa por la cual obtuvieron aprendizajes y cambios positivos (Cadena et al., 2013). 
También se ha demostrado que la autoestima es uno de los aspectos más importantes para el bienestar personal y para la interacción positiva con el entorno, que es una característica de resiliencia en pacientes con cáncer (Cerezo, 2014; Haase, 2004).

Entre1996 y 2002 se ha registrado un aumento significativo de casi 80\% en los niños y adolescentes que superan el cáncer (Ries et al., 2015). Este incremento ha motivado a los investigadores a estudiar las características psicosociales asociadas, y en los últimos años ha generado bastante interés por conocer la calidad de vida de esos jóvenes y de igual forma conocer las diferentes estrategias de afrontamiento que utilizaron con el objetivo de disminuir los efectos adversos físicos, cognitivos y psicológicos de la enfermedad y el tratamiento (Eshelman et al., 2004; Jacobs et al., 2009).

Siguiendo el planteamiento de los artículos científicos analizados al respecto, las preguntas de investigación que se pretenden responder son las siguientes: ¿cuáles son los factores de la resiliencia más prevalentes en una muestra de niños y adolescentes mexicanos que padecen cáncer? ¿Habrá diferencia en los niveles de resiliencia teniendo en cuenta el género y la edad?

Para responder estas cuestiones, se pretende identificar los principales factores de resiliencia en niños y adolescentes con cáncer, así como observar si existen diferencias significativas en cuanto a los niveles de resiliencia en género y edad.

Las hipótesis planteadas son que los participantes de menor edad tendrán mayores niveles de resiliencia que los mayores y que las mujeres presentarán mayores niveles de resiliencia que los hombres.

\section{Método}

\section{1. Participantes}

La muestra de estudio estuvo conformada por un total de 54 niños y adolescentes con cáncer, quienes provienen de dos asociaciones que les brindan apoyo. El porcentaje de hombres fue de $59.3 \%(N=32)$ y $40.7 \%(N=22)$ para mujeres, con una media de edad de 12.35 años $(D . T .=4.42) .{ }^{1}$

\section{2. Instrumentos}

En principio se utilizó una entrevista inicial para recabar datos sociodemográficos y clínicos, y para evaluar la resiliencia se empleó el Inventario de factores personales de resiliencia (Salgado, 2005). Esta escala consta de 48 ítems que evalúan cinco factores: autoestima, empatía, autonomía, humor y creatividad y presenta un coeficiente de fiabilidad de la escala

1. D. T.= Desviación Típica total de 0.96 en el estudio original. La validez de contenido de cada escala, medida por el coeficiente de Aiken para autoestima es de $V=0.90$, para empatía $V=0.99$, para autonomía $V=0.93$, para humor $V=0.97$ y para creatividad $V=0.94$. Los puntos de corte en autoestima son alto: 9-10; medio: 6-8, bajo: 1-5; en empatía: alto: 8-10, medio: 5-7, bajo: 1-4; en autonomía: alto: 8-10, medio: 4-7, bajo: 1-3; en humor: alto: 7-10, medio: 4-6, bajo: 1-3; para la creatividad: alto: 6-8, medio: 3-5, bajo: 1-2. Para la escala de resiliencia total, los puntos de corte son hasta 18 puntos que se encuentran en la categoría "muy bajo". De 19 a 23 puntos es "bajo", de 24 a 33 puntos es "promedio", de 34 a 42 puntos es categoría "alto" y mayor de 42 puntos es "muy alto".

\section{3. Procedimiento}

Para este trabajo se obtuvieron todos los permisos requeridos para la investigación en seres humanos y la aprobación por parte de un comité de la universidad. Se siguió los estándares éticos de la declaración de Helsinki de 1964.

Posteriormente, se reclutó la muestra de manera intencional de dos asociaciones que apoyan a niños y adolescentes con cáncer y se obtuvo el consentimiento informado de los padres o tutores de los niños; después se les aplicaron los cuestionarios a los niños en un único momento de evaluación.

Se utilizó la prueba de Shapiro-Wilk para contrastar el supuesto de normalidad, con un alpha de 0.05, donde se observó que la distribución cumple el supuesto de normalidad, ya que los datos obtenidos son mayores que 0.05 y de esta manera se acepta la hipótesis nula.

Además, se analizaron los datos mediante el programa estadístico SPSs, mediante la prueba $t$ de Student para comparar medias y la $r$ de Pearson para correlacionar variables cuantitativas.

Cabe destacar que este trabajo presenta un diseño de investigación cuantitativo de tipo descriptivo y comparativo con una muestra no probabilística.

\section{Resultados}

\section{1. Datos clínicos}

A $72.2 \%(N=39)$ de los participantes le diagnosticaron leucemia; $9.3 \%(N=5)$ algún tipo de tumor; 3.7\% $(N=2)$ histiocitosis; 3.7\% $(N=2)$ linfoma de Hodgkin; 1.9\% $(N=$ 1) metástasis; $1.9 \%(N=1)$ carcinoma y $1.9 \%(N=1)$ trombocitemia esencial.

En cuanto al tipo de tratamiento, a $59.3 \%(N=32)$ se les aplicó quimioterapia; 3.7\% $(N=2)$ tomaba otros medicamentos; $1.9 \%(N=1)$ radioterapia y $22.2 \%(N=12)$ se encontraba en estado de vigilancia. 


\section{2. Factores de resiliencia en niños y adolescentes con cáncer}

Los factores que se midieron con el instrumento utilizado en esta investigación fueron autoestima, empatía, autonomía, humor y creatividad (cuadro 1).

Los resultados demostraron que $70.4 \%$ de los participantes obtuvieron un nivel alto en el factor sentido del humor, por lo que se considera el factor de resiliencia más predominante en esta muestra de estudio.

El factor de empatía se encontró en 59.3\% de los participantes, la autonomía en $53.8 \%$, la autoestima en $53.7 \%$ y la creatividad en $31.5 \%$.

A modo de descripción general de la muestra, comparando las medias de cada subescala con los puntajes del instrumento original, se obtuvieron los siguientes resultados:

Como se puede observar, las variables de autoestima, empatía, autonomía y creatividad de la muestra están en el rango de puntaje medio; en cambio, las variables de humor y resiliencia total en el rango alto.

\section{3. Resiliencia y género}

En cuanto a las diferencias en resiliencia respecto al género, los resultados mostraron que sí existen diferencias estadísticamente significativas entre hombres y mujeres en: autoestima $(t=-27.832 ; p<0.01)$, empatía $(t=-25.590 ; p<0.01)$, autonomía $(t=-24.078 ; p<0.01)$, humor $(t=-19.654 ; p<0.01)$, creatividad $(t=-15.762 ; p<0.01)$ y en resiliencia total $(t=$ -43.723 ; $p<0.01)$. Se observa que los hombres tienen niveles más altos en los factores de autoestima, empatía, autonomía, sentido del humor y resiliencia total; en cambio, las mujeres obtuvieron un resultado más alto en el factor de creatividad.

\section{4. Resiliencia y edad}

Los resultados mostraron que existe una correlación positiva moderada entre la edad y la autoestima $(r=0.386, p<0.01)$, así como en la empatía $(r=0.398, p<0.01)$, la autonomía $(r$ $=0.383, p<0.01)$ y la resiliencia total $(r=0.387, p<0.01)$.

Sin embargo, no se obtuvieron diferencias estadísticamente significativas en los factores de sentido del humor $\mathrm{y}$ de creatividad (ambos con $p>0.05$ ).

\section{Discusión}

El objetivo fue estudiar la resiliencia en niños y adolescentes con cáncer para conocer los factores que están presentes en esta población y observar si existen diferencias en función del sexo y la edad.
En primer lugar se puede observar que los factores de resiliencia propuestos por Salgado (2005) sí estaban presentes en la muestra de estudio.

En este tenor, se vio que los factores más predominantes fueron de mayor a menor: sentido del humor (70.4\%), empatía (59.3\%), autonomía (53.8\%), autoestima (53.7\%) y creatividad $(31.5 \%)$.

Estos resultados además coinciden con los resultados de otras investigaciones analizadas (Bragado, 2010; Fredrickson y Tugade, 2003; González-Arratia, 2009).

El factor más común en los participantes fue el sentido del humor; por tanto, la capacidad de ver el lado cómico de las situaciones desagradables es importante para desarrollar la resiliencia y es probable que los pacientes utilicen este tipo de emociones positivas como forma de afrontar el cáncer (Fredrickson y Tugade, 2003). En cambio, la creatividad fue el factor menos popular presente en los niños y adolescentes con cáncer encuestados en este estudio. Los factores autonomía, empatía y autoestima se manifestaron en más de la mitad de los participantes.

En segundo lugar, en relación con el género, los resultados obtenidos refutan parcialmente los resultados de otros estudios. Sí se encontraron diferencias estadísticamente significativas en resiliencia en función del género; sin embargo, al contrario de estudios anteriores donde se muestra que la resiliencia es mayor en las mujeres (González-Arratia et al., 2009; Morales y Díaz, 2011), se encontró que los factores de empatía, autoestima, autonomía y sentido del humor son mayores en los hombres y el factor creatividad en mujeres. Con esto, la hipótesis planteada se acepta de manera parcial.

En tercer lugar se observan correlaciones positivas y significativas entre la edad y la resiliencia en los factores de autoestima, empatía y la autonomía. Es decir, a mayor edad, existen mayores niveles de estos tres factores analizados. Estos resultados son contrarios a estudios anteriores, donde se observó que la resiliencia era mayor en niños más pequeños

\begin{tabular}{llc} 
Cuadro 1. & Comparación de puntuaciones. \\
\hline \multicolumn{1}{c}{$\begin{array}{c}\text { Puntos de corte de la escala original } \\
\text { (Salgado, 2005) }\end{array}$} & $\begin{array}{c}\text { Medias obtenidas de } \\
\text { la muestra }\end{array}$ \\
\hline Autoestima & alto: 9 -10; medio: 6 -8; bajo: $1-5$ & 8.30 \\
Empatía & alto: $8-10$; medio: 5 -7; bajo: $1-4$ & 7.70 \\
Autonomía & alto: $8-10$; medio: $4-7$; bajo: $1-3$ & 7.44 \\
Humor & alto: $7-10$; medio: $4-6$; bajo: $1-3$ & 7.19 \\
Creatividad & alto: $6-8$; medio: $3-5$; bajo: $1-2$ & 4.67 \\
Resiliencia total & $<18$ puntos muy bajo; $19-23$ bajo; 24-33 promedio; & 35.30 \\
& $34-42$ alto; $>42$ muy alto & \\
\hline
\end{tabular}


(Bragado, 2010). Por el contrario, no existe relación entre el sentido del humor, la creatividad y la edad. Así pues, la hipótesis planteada de que los niños más pequeños tendrán mayores niveles de resiliencia, queda rechazada.

El interés que suscita el estudio de la resiliencia en esta población es que es un aspecto que ayuda a encontrar un equilibrio emocional y psicológico que puede ser muy importante para que los niños y adolescentes con cáncer afronten su enfermedad y logren recuperarse sin tener consecuencias psicológicas negativas graves.

Las limitaciones enfrentadas hacen referencia al tamaño reducido de la muestra, el tipo de muestreo no probabilístico y que no se tienen en cuenta estresores ambientales a parte de la enfermedad, así como tampoco aspectos familiares y sociales. Por consiguiente, sería enriquecedor en futuros trabajos estudiar más a fondo estas variables, sobre todo el papel de la familia y el apoyo social, ya que otros autores las observan como parte fundamental y factor resiliente (Blum, 1997; González-Arratia, 2009). Esto serviría para conocer el ambiente familiar, los roles que ejercen dentro de él, las reglas de funcionamiento, la permeabilidad de los límites y el apoyo social y familiar que perciben los niños y adolescentes con cáncer.

Como conclusión, el artículo pretende un acercamiento al estudio empírico de la resiliencia en niños y adolescentes con cáncer. Este nuevo enfoque de estudiar las cualidades o factores resilientes en esta población surge de la necesidad de adaptar los tratamientos psicológicos y de prevenir posibles trastornos psicológicos en un futuro. Por este motivo, es sumamente útil en términos prácticos atender esta problemática de un modo novedoso y potencializar las cualidades positivas presentes en los pacientes, ya que es bien conocido que el bienestar emocional en estos pacientes ayuda a aumentar el sistema inmune. Por el contrario, el malestar emocional y los síntomas adyacentes como los depresivos y ansiosos influyen de manera negativa en el desarrollo del sistema inmune.

\section{Análisis prospectivo}

Tenemos ante nosotros la oportunidad de buscar más allá de la patologización de enfermedades crónicas, como es el caso del cáncer, y analizar los factores que pueden ayudar a las personas a salir adelante e incluso a crecer después de un evento estresante, ya que si pudiésemos trabajar en el desarrollo de la resiliencia en niños y adolescentes con cáncer podríamos fortalecer el aspecto psicológico con el que enfrentan el proceso de su enfermedad.

Los resultados arrojados son novedosos en cuanto al estudio de la resiliencia en niños y adolescentes con cáncer en México; sin embargo, es necesario atender a las limitaciones para aumentar el rigor científico con el que se pretende trabajar $\mathrm{y}$ no solamente quedarnos en un nivel descriptivo de los resultados. Para ello, teniendo en cuenta el análisis prospectivo, la mira a futuro consistiría en obtener una muestra más amplia de participantes para conseguir datos más confiables; asimismo, utilizar otro tipo de escala que mida la resiliencia para complementar la aplicada. Incluso, se sugiere elaborar una entrevista clínica inicial para lograr datos cualitativos de cada participante y aumentar la información de cada paciente para obtener datos más completos, así como analizar los estresores ambientales, el ambiente familiar y el apoyo social percibido mediante instrumentos de evaluación específicos.

De este modo, la principal dirección a futuro es analizar con mayor profundidad dichas fortalezas, estudiar más a fondo variables como el entorno familiar y finalmente, con los resultados obtenidos, proponer intervenciones específicas basadas en las fortalezas para mejorar la calidad de vida de los niños y adolescentes con cáncer.

Referencias

Álamo, G., Caldera, F., Cardozo, M. J. y González, Y. (2012). Resiliencia en personas con cáncer. Revista Arbitrada Formación Gerencial, 11(2), 270-292.

Allen, R., Newman, S. P. y Souhami, R. L. (1997). Anxiety and depression in adolescent cancer: Findings in patients and parents at the time of diagnosis. European
Journal of Cancer, 33, 1250-1255.

Becoña, E. (2006). Resiliencia: definición y características y utilidad del concepto. Revista de Psicopatología y Psicología Clínica, 11(3), 125-146.

Blum, R. (1997). Riesgo y resiliencia. Conceptos básicos para el desarrollo de un programa. Adolescencia Latinoamericana, 1(1), 16-19.
Bonanno, G. (2004). Loss, trauma and human resilience. American Psychologist, 20-28.

Bowen, D., Morasca, A. y Meischke, H. (2003). Measures and correlates of resilience. Women y Health, 38(2), 65-75.

Bragado, C. (2010). Ajuste psicológico y resiliencia en niños y adolescentes con cáncer. Consejo General de Colegios 
Oficiales de Psicológos. INFOCOP, 47, 37-38.

Cabanyes, J. (2010). Resiliencia: una aproximación al concepto. Revista de Psiquiatría y Salud Mental, 3(4), 145-151.

Cadena, A., Castro, G. y Franklin, E. (2013). Vivencias y significados de adolescentes sobrevivientes al cáncer. Trilogía. Ciencia, Tecnología y Sociedad, 10, 145-163.

Castro, G. y Moreno-Jiménez (2007). Resiliencia en niños enfermos crónicos: aspectos teóricos. Psicologia em Estudo, 12(1), 81-86.

Cedillo, M. (2014) Resiliencia en niños con problemas oncológicos. Toluca. Disponible en http://hdl.handle.net/10401/6566

Cerezo, G. (2014). Variables psicológicas positivas en pacientes con cáncer. Información psicológica, 106, 17-27. DOI: http://dx.medra.org/10.14635/IPSIC.2014.106.2

Chaves, C. (2014). Emociones positivas y bienestar en niños con enfermedades crónicas (tesis doctoral).

Dejong, M. y Fombonne, E. (2006). Depression in pediatric cancer: An overview. Psycho-Oncology, 15, 553-566.

Eiser, C., Hill, J. J. y Vance, Y. H. (2000). Examining the psychological consequences of surviving childhood cancer: Systematic review as a research method in pediatric psychology. Journal of Pediatric Psychology, 25, 449-460.

Eshelman, D., Landier, W., Sweeney, T., Hester, A., Forte, K., Darling, J. y Hudson, M. (2004). Facilitating care for childhood cancer survivors: Integrating children's oncology group long-term follow-up guidelines and health links in clinical practice. Journal Pediatric Oncology Nurs, 5, $271-280$

Espada, J., López-Roig, S., Méndez, J. y Orgilés, M. (2004). Atención psicológica en el cáncer infantil. Dialnet, 1(1), 139-154.
Fraser, M. W., Rischman, J. M. y Galinsky, M. J. (1999). Risk, protection and resilience: Toward a conceptual framework for social work practice. Social Work Research, 23(3), 131-143.

Fredrickson, L. B. y Tugade, N. M. (2003). Good are positive emotions in crises? A prospective study of resilience and emotions following the terrorist attacks on the USA on September 11 th. 2001. Journal of Personality and Social Psychology, 2.

García, J. (2013). Efecto de un programa cognitivo-conductual en la resiliencia en niños con cáncer (tesis de maestría).

González-Arratia, N. (2009). Resiliencia y salud en niños y adolescentes. CIENCIA ergo-sum, 16(3), 247-253.

González-Arratia, N., Nieto, D. y Valdez, J. (2011). Resiliencia en madres e hijos con cáncer. Psicooncología, 8(1), 113-123.

González-Arratia, N., Valdez-Medina, J. y Zavala, Y. (2008). Resiliencia en adolescentes mexicanos. Enseñanz̧ e investigación en Psicología, 13(1), 41-52.

González-Arratia, N., Valdez, J., Oudhof, H. y González, S. (2009). Resiliencia y salud en niños con cáncer. CIENCIA ergo-sum, 16, 247-253.

Grootenhuis, M. A. y Last, B. F. (2001). Children with cancer with different survival perspectives: Defensiveness, control strategies and psychological adjustment. Psycho-Oncology, 10, 305-314.

Grotberg, E. (1995). Una guía para promover la resiliencia en niños: fortaleciendo el espiritu bumano, proyecto internacional de la resiliencia. La Haya: Bernard Van Leer Fundation.

Grotberg, E. (2006). La resiliencia en el mundo de hoy: cómo superar las adversidades. España: Gedisa Editorial.

Haase, J. (2004). The adolescent resilience model as a guide to interventions. Journal of Pediatric Oncology Nursing, 21(5), 289-299.
IMSS (Instituto Mexicano del Seguro Social) (2011). El registro de cáncer en niños, fundamental para prevenir, atender y controlar la enfermedad. Revista Médica del Instituto Mexicano del Seguro Social, 49(1), 33-38.

Jacobs, L. A., Palmer, S. C., Schwartz, L. A., DeMichele, A., Mao, J. J., Garver, J., García, C., Hill-Kayser, C. E., Metz, J. M., Margaret Klampshire, K., Va chani, C., Pucci, D. y Meadows, A. T. (2009). Adult cancer survivorship: Evolution, research, and planning care. CA cancer Journal for Clinicians, 59, 391-410.

Manciaux, M. (2003). La resiliencia: resistir y rehacerse. Madrid: Gedisa Editorial.

Manciaux, M., Vanistendael, S., Lecomte, J. y Cyrulnik, B. (2001). La resiliencia: estado de la cuestión, en M. Manciaux (ed.), La resiliencia: resistir y rehacerse. Madrid: Gedisa Editorial.

Masten, A. y Reed, M.G. (2002). Resilience in development. Handbook of positive Psychology, 74-88.

Masten, A. S. y Powell, J. L. (2003). A resilience framework for research, policy, and practice, en S. S. Luthar (ed.), Resilience and vulnerability: Adaptation in the context of childhood adversities (pp. 1-29). Cambridge: University Press.

Melillo, A., Suárez, E. y Rodríguez, D. (2004). Resiliencia y subjetividad. Paidós: Argentina.

Morales, M. y Díaz, D. (2011). Estudio comparativo de la resiliencia en adolescentes: el papel del género, la escolaridad y procedencia. Uaricha, 8(17), 62-77.

Muñoz, G. (2005). Educar para la resiliencia. Un cambio de mirada en la prevención de situaciones de riesgo social. Revista Complutense de Educación, 16(1), 107-124.

Mystakidou, K., Tsilika, E., Parpa, E., Katsouda, E., Sakkas, P., Galanos, A. y Vlahos, L. (2006). Demographic and clinical predictors of preparatory grief 
in a sample of advanced cancer patients.

Psycho-oncology, 15, 828-833.

Noll, R. B., Garstein, M. A., Vannatta, K., Correll, J., Bukowski, W. M. y Davies, W. H. (1999). Social, emotional and behavioral functioning of children with cancer. Pediatrics, 103, 71-78.

Phipps, S. (2007). Adaptive style in children with cancer: Implications for a positive psychology approach. Journal of Pediatric Psychology, 32(9), 1055-1066.

Place, M., Reynolds, J., Cousins, A. y O’Neill, S. (2002). Developing a resilience package for vulnerable children. Child and Adolescent Mental Health, 7(4), 162-167.

Prado, R. y Águila, M. (2003). Diferencia en la resiliencia según género y nivel socioeconómico en adolescentes. Dialnet, 179-196.

Puig, G. y Rubio, J. L. (2011). Manual de resiliencia aplicada. Barcelona: Gedisa Editorial
Ries, L., Harkins, D., Krapcho, M., Mariotto, A., Miller, B. A., Feuer, E. J., Clegg, L., Eisner, M. P, Horner, M. J., Howlader, N., Hayat, M., Hankey, B. F., Edwards, B. K. (eds.). (2015). SEER cancer statistics review, 1975-2003. National Cancer Institute.

Romero, S. (1998). Resiliencia: ¿enemigo o aliado para el desarrollo humano?. Documentos CIDE, 9.

Rutter, M. (1992). Developing minds: Challenge and continuity across the life span. United Kingdow: Penguin Books.

Saavedra, E. y Villalta, M. (2008). Medición de las características resilientes: un estudio comparativo en personas entre $15 \mathrm{y}$ 65 años. Scielo, 14(14).

Salgado, A. (2005). Inventario de resiliencia para niños: fundamentación teórica y construcción. Cuaderno de Investigación 8. Lima: Universidad de San Martín de Porres.

Salgado, A. (2005). Métodos e instrumentos para medir la resiliencia: una alternativa peruana. Liberabit, 11(11), 41-48.
Stein, H., Fonagy, P., Ferguson, K. S. y Wisman, M. (2000). Lives through time: An ideographic approach to the study of resilience. The bulletin of the Menninger Clinic, 64(2), 281-305.

Suárez, E. y Krauskorpf, D. (1995). El enfoque de riesgo y su aplicación a las conductas del adolescente. Una perspectiva psicosocial. Publicaciones científicas, 552, ops/OMs.

Tarragona, M. (2011). La resiliencia: el resorte del bienestar. Prensa, 1, 1-13.

Vázquez, C. y Castilla, C. (2007). Emociones positivas y crecimiento postraumático en el cáncer de mama. Psicooncología, 4, $385-404$

Von Essen, L., Enskär, K., Kreuger, A., Larsson, B. y Sjödén, P. O. (2000). Selfesteem, depression and anxiety among swedish children and adolescents on and off treatment. Acta Pediátrica, 89, 229-236.

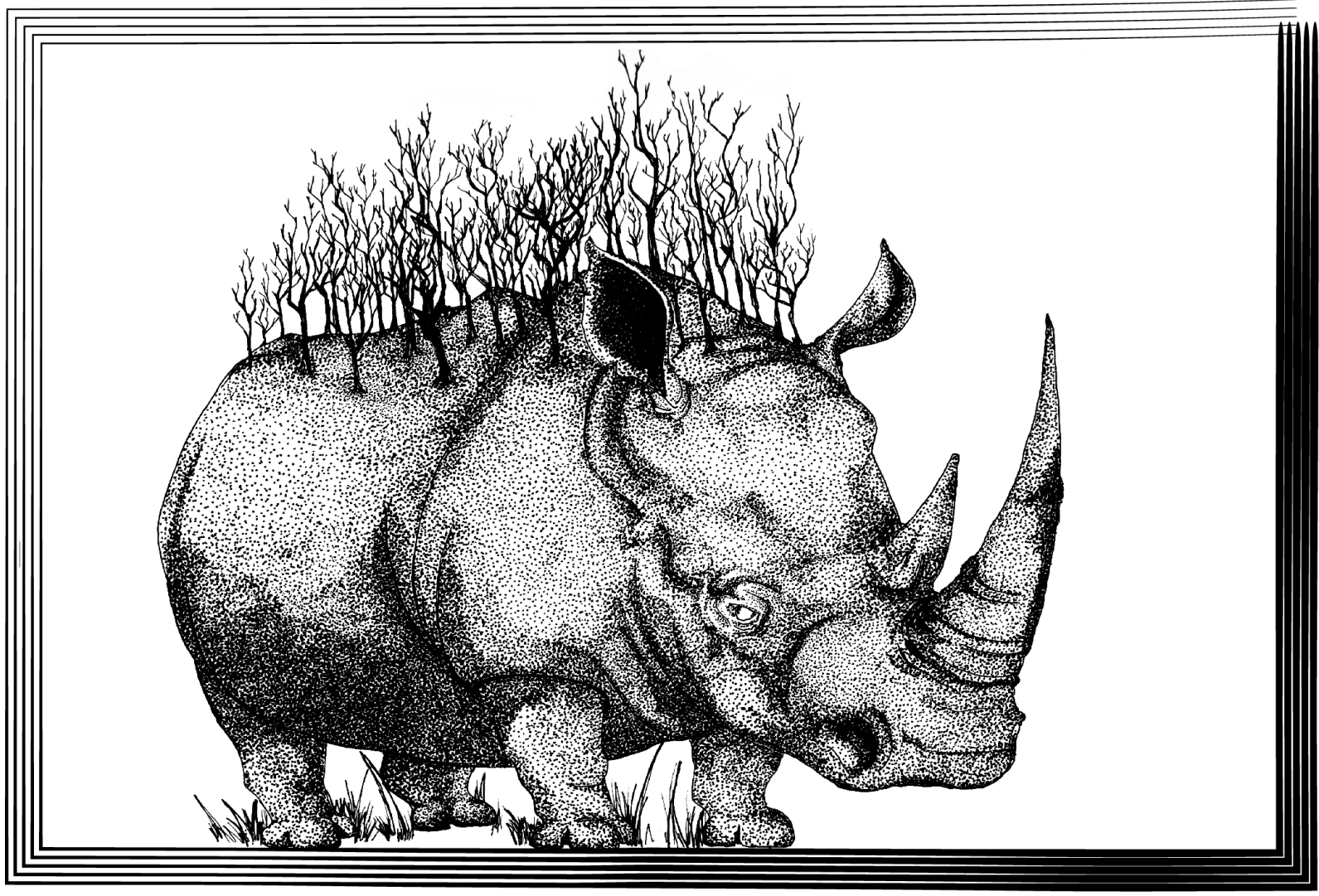

Ilustración: Bettina Montes de Oca Corral 